\title{
A European regulatory perspective on cystic fibrosis: current treatments, trends in drug development and translational challenges for CFTR modulators
}

\author{
Stefano Ponzano ${ }^{1,2}$, Giulia Nigrelli ${ }^{1}$, Laura Fregonese ${ }^{1}$, Irmgard Eichler ${ }^{1}$, \\ Fabio Bertozzi², Tiziano Bandiera ${ }^{2}$, Luis J.V. Galietta ${ }^{3}$ and Marisa Papaluca ${ }^{1}$ \\ Affiliations: ${ }^{1}$ European Medicines Agency, London, UK. ${ }^{2}$ D3 PharmaChemistry, Istituto Italiano di Tecnologia, \\ Genova, Italy. ${ }^{3}$ Telethon Institute of Genetics and Medicine, Pozzuoli, Italy.
}

Correspondence: Stefano Ponzano, Clinical Pharmacology and Non-clinical Support Office, Specialised Scientific Disciplines Dept, European Medicines Agency, 30 Churchill Place, Canary Wharf, London, E14 5EU, UK. E-mail: stefano.ponzanodema.europa.eu

@ERSpublications

Alternative nonclinical models for CFTR modulators are needed to increase prediction of clinical efficacy http://ow.ly/Tj8y30iQNiD

Cite this article as: Ponzano S, Nigrelli G, Fregonese L, et al. A European regulatory perspective on cystic fibrosis: current treatments, trends in drug development and translational challenges for CFTR modulators. Eur Respir Rev 2018; 27: 170124 [https://doi.org/10.1183/16000617.0124-2017].

ABSTRACT In this article we analyse the current authorised treatments and trends in early drug development for cystic fibrosis (CF) in the European Union for the time period 2000-2016. The analysis indicates a significant improvement in the innovation and development of new potential medicines for CF, shifting from products that act on the symptoms of the disease towards new therapies targeting the cause of CF. However, within these new innovative medicines, results for CF transmembrane conductance regulator (CFTR) modulators indicate that one major challenge for turning a CF concept product into an actual medicine for the benefit of patients resides in the fact that, although pre-clinical models have shown good predictability for certain mutations, a good correlation to clinical end-points or biomarkers (e.g. forced expiratory volume in $1 \mathrm{~s}$ and sweat chloride) for all mutations has not yet been achieved. In this respect, the use of alternative end-points and innovative nonclinical models could be helpful for the understanding of those translational discrepancies. Collaborative endeavours to promote further research and development in these areas as well as early dialogue with the regulatory bodies available at the European competent authorities are recommended.

\section{Introduction}

Cystic fibrosis (CF) is the most common autosomal recessive disease among Caucasians and is estimated to affect about 36000 individuals in the European Union (EU) [1]. Approximately 80000 people in the world are diagnosed with CF [2], showing wide geographic and ethnic variations, with an incidence of 1 in 2500 Caucasians (the highest incidence is found in the Republic of Ireland, while in Finland CF is extremely rare) [2-4]. CF is rare in persons of non-Caucasian origin.

$\mathrm{CF}$ is caused by mutations in the CF transmembrane conductance regulator (CFTR) protein, a cAMP-regulated chloride channel expressed primarily at the apical plasma membrane of secretory

This article has supplementary material available from err.ersjournals.com

Received: Nov 062017 | Accepted after revision: Feb 142018

Provenance: Submitted article, peer reviewed.

Copyright CERS 2018. ERR articles are open access and distributed under the terms of the Creative Commons Attribution Non-Commercial Licence 4.0. 
epithelia in the airways, pancreas, intestine and other organs. CFTR is a member of the ATP-binding cassette transporter superfamily, which differs from other members not only in its structure [5], but primarily in the function, CFTR being the only ion channel [6-8]. The basic defect due to CFTR mutations results in poor chloride and bicarbonate transport, which causes dehydration of secretions with viscous mucus and leads to different, severe pathologies, such as inflammation and chronic airway obstruction, pancreatic and digestive insufficiency, bowel obstruction, diabetes, hepatic damage, and male infertility [9-13]. While many organs are affected in CF, pulmonary disease is the major cause of morbidity and mortality. These features are the hallmarks of CF and result in limited life expectancy [14, 15].

Almost 2000 mutations in the CFTR gene have been identified that can produce a reduction or loss-of-function phenotype by impairing its translation, cellular processing and/or chloride channel gating [16]. CF mutations have been grouped and classified into six categories (class I-VI) according to the different mechanism by which they are known to disrupt CFTR production and function (table 1) [17-20]. Class I mutations include nonsense mutations (e.g. G542X and W1282X), generating premature termination codons that lead to truncated nonfunctional protein. Class II mutations have little or no detectable CFTR at the plasma membrane as a consequence of misfolding of the newly translated protein. This category includes the most common mutation, the deletion of phenylalanine 508 (F508del), present in one or both alleles in $\sim 90 \%$ of CF patients [2]. F508del CFTR mutation results in misfolded CFTR channels that are predominantly recognised and degraded by the endoplasmic reticulum quality control system $[21,22]$. Both class I and II impair CFTR protein production and plasma membrane expression, causing a severe CF phenotype. Class III (e.g. G551D, expressed in $\sim 4-5 \%$ of CF patients) and class IV (e.g. R117H, R334W and R347P) mutations impair CFTR channel gating and conductance, respectively, without significant trafficking defects $[2,8,23,24]$. Class $\mathrm{V}$ mutations cause mRNA mis-splicing or interfere with the promoter activity, whereas class VI mutations reduce CFTR stability, leading to accelerated channel removal from the plasma membrane $[8,25]$.

Clinically, CF manifests as a systemic disease showing variable severity and a broad clinical spectrum depending on the target organs and systems mostly affected [8-10]. One of the pathological hallmarks of classical CF $[26,27]$, with impaired chloride and bicarbonate secretion, is characterised by accumulation of dehydrated, acidified and hyperviscous mucus in the lungs that compromises mucociliary clearance and bacterial killing [28]. This makes CF airways vulnerable to recurring infections and permanent inflammation, leading to airway obstruction/destruction, respiratory failure and death [10]. Structural bronchopulmonary changes occur much earlier in life than previously thought and can be detected shortly after diagnosis in CF newborns, as part of the European newborn screening in CF [29-31]. Recurrent exacerbations, typically triggered by acute bacterial infections, promote progressive loss of lung tissue architecture with bronchiectasis.

The majority of CF patients $(\sim 85 \%)$ have pancreatic exocrine insufficiency, even in early infancy, due to ductal obstruction, which could lead to fat malabsorption and malnutrition [32]. Early gastrointestinal manifestations include meconium ileus in the newborn patients (10-17\%). Sweat glands are most consistently affected in classical CF, and produce sweat with elevated chloride $\left(>60 \mathrm{mmol} \cdot \mathrm{L}^{-1}\right)$ and sodium concentrations [9]. Moreover, $\sim 98 \%$ of CF males are infertile because of atrophic or absent vasa deferentia [8].

\begin{tabular}{|c|c|c|}
\hline Class & Mutation examples & Mutation mechanism \\
\hline I & G542X, W1282X & $\begin{array}{c}\text { No functional CFTR protein is synthesised due to the presence of } \\
\text { premature termination codons }\end{array}$ \\
\hline II & F508del & $\begin{array}{c}\text { CFTR protein is synthesised but misfolded, keeping it from reaching } \\
\text { the apical cell surface }\end{array}$ \\
\hline III & G551D, G1349D & $\begin{array}{c}\text { Full-length CFTR protein reaching the apical cell surface exhibits } \\
\text { abnormal gating (channel persists in the closed state) }\end{array}$ \\
\hline IV & R117H, R334W, R347P & $\begin{array}{l}\text { Full-length CFTR protein reaches the apical cell surface but } \\
\text { transport of chloride ions is reduced due to channel pore defect }\end{array}$ \\
\hline v & $2789+5 G>A, 3849+10 k b C>T$ & $\begin{array}{l}\text { Reduced number of CFTR transcripts due to promoter or splicing } \\
\text { abnormalities }\end{array}$ \\
\hline VI & 4326delTC, 4279insA & $\begin{array}{c}\text { Defective CFTR stability at the cell surface; C-terminus mutations } \\
\text { result in accelerated turnover }\end{array}$ \\
\hline
\end{tabular}

CFTR: CF transmembrane conductance regulator. 


\section{Current therapies for CF}

When CF was first described in 1938 by Dorothy Andersen [33], the predicted survival age of a CF patient was only 6 months. For patients born in the 1990s, median survival is now predicted to exceed 40 years $[34,35]$, with lung disease as the principal cause of morbidity and mortality. This significant gain in life expectancy is the result of advances in early diagnosis and symptomatic treatments based on vast improvements in nutrition, control of airway infections and physiotherapy. However, despite great advances in supportive care and in our understanding of the pathophysiology of the disease, treatment options that effectively halt progression of the disease and may extend life expectancy are currently limited to a small minority of relatively rare CFTR mutations [36].

Subsequent to the involvement of the European Medicines Agency (EMA) in medicines for rare diseases (2000), nine products were approved for CF treatment within the centralised procedure up to December 2016 (table 2). CFTR modulators represent the most promising innovation in CF therapy as they target the cause of the disease, whereas the other authorised products provide only symptomatic treatments and include antibiotics, osmotic agents and pancreatic enzyme products.

\section{Antibiotics in CF}

Inhaled and systemic (oral and/or intravenous) antibiotic treatment of chronic bacterial infections is still considered the basis for CF therapy, strongly contributing to the substantially increased life expectancy of patients in recent decades.

TABLE 2 Cystic fibrosis (CF) products that received a marketing authorisation within the centralised procedure until 2016

\begin{tabular}{|c|c|c|c|c|c|}
\hline $\begin{array}{r}\text { Medicinal } \\
\text { product }\end{array}$ & $\begin{array}{l}\text { Active } \\
\text { substance }\end{array}$ & $\begin{array}{l}\text { Date of } \\
\text { authorisation }\end{array}$ & $\begin{array}{l}\text { Marketing authorisation } \\
\text { holder }\end{array}$ & Approved indication & $\begin{array}{l}\text { Orphan } \\
\text { medicinal } \\
\text { product }^{\#}\end{array}$ \\
\hline $\begin{array}{l}\text { TOBI } \\
\text { Podhaler }\end{array}$ & Tobramycin & July 20, 2011 & $\begin{array}{l}\text { Novartis Europharm Ltd, } \\
\text { Camberley, UK }\end{array}$ & $\begin{array}{l}\text { Suppressive therapy of chronic pulmonary } \\
\text { infection due to } P \text {. aeruginosa in adults and } \\
\text { children with CF aged } \geqslant 6 \text { years }\end{array}$ & Yes \\
\hline Colobreathe & $\begin{array}{l}\text { Colistimethate } \\
\text { sodium }\end{array}$ & Feb 13, 2012 & $\begin{array}{l}\text { Teva BV, Haarlem, } \\
\text { The Netherlands }\end{array}$ & $\begin{array}{l}\text { Management of chronic pulmonary infections } \\
\text { due to } P \text {. aeruginosa in patients with CF aged } \\
\qquad 6 \text { years }\end{array}$ & No \\
\hline Bronchitol & Mannitol & April 13, 2012 & $\begin{array}{l}\text { Pharmaxis Pharmaceuticals } \\
\text { Ltd, London, UK }\end{array}$ & $\begin{array}{l}\text { Treatment of CF in adults aged } \geqslant 18 \text { years as an } \\
\text { add-on therapy to best standard of care }\end{array}$ & Yes \\
\hline Kalydeco & Ivacaftor & July 23, 2012 & $\begin{array}{l}\text { Vertex Pharmaceuticals } \\
\text { (Europe) Ltd, London, UK }\end{array}$ & $\begin{array}{l}\text { Treatment of CF in patients aged } \geqslant 2 \text { years who } \\
\text { have the following mutations in the CFTR gene: } \\
\text { G551D, G1244E, G1349D, G178R, G551S, } \\
\text { S1251N, S1255P, S549N and S549R; it is also } \\
\text { indicated for the treatment of adult CF patients } \\
\text { who have an R117H mutation in the CFTR gene }\end{array}$ & Yes \\
\hline Vantobra & Tobramycin & $\begin{array}{l}\text { March 18, } \\
2015\end{array}$ & $\begin{array}{l}\text { PARI Pharma GmbH, } \\
\text { Starnberg, Germany }\end{array}$ & $\begin{array}{l}\text { Management of chronic pulmonary infection } \\
\text { due to } P \text {. aeruginosa in patients with CF aged } \\
\geqslant 6 \text { years }\end{array}$ & No \\
\hline Quinsair & Levofloxacin & $\begin{array}{l}\text { March 26, } \\
2015\end{array}$ & $\begin{array}{l}\text { Chiesi Orphan BV, Schiphol, } \\
\text { The Netherlands }\end{array}$ & $\begin{array}{l}\text { Management of chronic pulmonary infections } \\
\text { due to } P \text {. aeruginosa in adult patients with } C F\end{array}$ & No \\
\hline Orkambi & $\begin{array}{l}\text { Lumacaftor/ } \\
\text { ivacaftor }\end{array}$ & Nov 19, 2015 & $\begin{array}{l}\text { Vertex Pharmaceuticals } \\
\text { (Europe) Ltd, London, UK }\end{array}$ & $\begin{array}{c}\text { Treatment of CF in patients aged } \geqslant 12 \text { years } \\
\text { who are homozygous for the F508del mutation } \\
\text { in the CFTR gene }\end{array}$ & No \\
\hline Enzepi ${ }^{\Uparrow}$ & $\begin{array}{l}\text { Pancreas } \\
\text { powder }\end{array}$ & June 29, 2016 & $\begin{array}{l}\text { Allergan Pharmaceuticals } \\
\text { International Ltd, Dublin, } \\
\text { Ireland }\end{array}$ & $\begin{array}{c}\text { Pancreatic enzyme replacement treatment in } \\
\text { exocrine pancreatic insufficiency due to CF or } \\
\text { other conditions (e.g. chronic pancreatitis, } \\
\text { post-pancreatectomy or pancreatic cancer); } \\
\text { indicated in adults, infants, children and } \\
\text { adolescents }\end{array}$ & No \\
\hline
\end{tabular}

P. aeruginosa: Pseudomonas aeruginosa. ": for more details about the definition of orphan designation, see section "Trends in CF drug development"; ": Enzepi was withdrawn from the market in July 2017 for commercial reasons. 
Cayston was the first CF medicine to be approved by the EMA in 2009 and is indicated for the chronic pulmonary infection due to Pseudomonas aeruginosa, the major pathogen in CF [37]. The active substance in Cayston, aztreonam, is a monobactam antibiotic belonging to the " $\beta$-lactam" class [38], available as an injection since the 1980s in the form of an arginine salt, but in Cayston it is formulated as a lysine salt in a solution that can be nebulised and inhaled directly into the lungs.

Since the introduction of Cayston, four other antibiotics have been approved by the EMA (table 2) for the treatment of chronic pulmonary infections due to $P$. aeruginosa. These drugs contain well-known active principles that are able to either disrupt the bacterial cell wall (tobramycin (TOBI Podhaler and Vantobra) and colistin (Colobreathe)) or inhibit specific enzymes that the pathogen uses for self-replication (levofloxacin (Quinsair)). Type of formulation, droplet and particle size, drug output rate, and distribution of the drug throughout the infected area as well as the type of device used for nebulisation and inhalation are determinant factors for the overall efficacy of the product [39].

In general, inhalation of antibiotic aerosols (wet and dry) allows topical delivery and results in peak concentrations at the infection site (airways lumen) that are orders of magnitude higher than those achieved by i.v. or oral administration [40,41]. Furthermore, the systemic exposure following absorption of the inhaled active substance from the lung results in peak serum drug levels that are substantially lower than those observed with systemic antipseudomonal antibiotic treatments [42]. These factors should in principle make the new inhaled antibiotics safer and more efficacious compared with earlier treatments (i.v. and oral), which possess dose-limiting systemic toxicities [18, 37]. According to a Cochrane review, a pooled estimate of the level of benefit from the use of inhaled antibiotics as a standard for CF patients infected chronically with $P$. aeruginosa is still not possible from the available data [43].

Osmotic agents in CF

In 2012, the EMA recommended the approval of a new product for inhalation, Bronchitol, containing mannitol as an active substance that increases the surface liquid in the airways. This is due to an osmotic gradient that favours movement of water into the lumen, thereby enhancing mucociliary clearance [44]. In phase 3 pivotal clinical trials, inhaled mannitol resulted in a modest but statistically significant improvement of lung function relative to control, as measured by forced expiratory volume in $1 \mathrm{~s}$ (FEV1), forced vital capacity and the incidence of having a protocol-defined pulmonary exacerbation. The product showed an overall good safety profile over 26 weeks of treatment $[45,46]$.

\section{CFTR modulators}

Since the identification of the genetic cause of CF and the mutations in the gene coding for CFTR protein, great efforts have been made to develop agents that could target the disease at the CFTR level by improving the deficient or defective activity of the mutated protein [47]. This strategy was further supported by the fact that $\mathrm{CF}$ is a monogenic disease and the molecular phenotypes of the most prevalent mutations are known [48].

Ivacaftor (Kalydeco) was approved in 2012 as the first authorised product targeting CFTR, followed by the combination lumacaftor/ivacaftor (Orkambi) 3 years later. These compounds belong to the class of CFTR modulators, further classified as potentiators and correctors depending on their action on CFTR. Ivacaftor, the active substance of Kalydeco, is a potentiator that acts by increasing the open channel probability of the CFTR channel, thus enhancing the chloride ion flow through the CFTR at the apical surface of cells $[49,50]$. Kalydeco is particularly suited for gating (class III) mutations; it is currently authorised by the EMA for the treatment of patients aged $\geqslant 2$ years with G551D, the main gating mutation, accounting for $\sim 4-5 \%$ of the CF patient population [23], and an additional eight other gating mutations (G1244E, G1349D, G178R, G551S, S1251N, S1255P, S549N and S549R). Kalydeco is also indicated for the treatment of adult CF patients who have an R117H mutation in the CFTR gene. In Orkambi, lumacaftor (a "corrector") improves intracellular trafficking of the mutant CFTR, allowing the protein to reach the apical surface $[49,51]$, where the ivacaftor component is able to enhance the channel function. Orkambi is currently authorised by the EMA for the treatment of CF patients aged $\geqslant 12$ years homozygous for the F508del mutation.

\section{Pancreatic enzyme products}

Enzepi was approved by the EMA in 2016 for the treatment of exocrine pancreatic insufficiency due to $\mathrm{CF}$ or other conditions (e.g. chronic pancreatitis, post-pancreatectomy or pancreatic cancer). The product is a pancreatic enzyme product containing pancreas powder, an extract derived from porcine pancreatic glands, which includes multiple enzyme classes such as lipases, proteases and amylases. The approved medicine catalyses the hydrolysis (in the duodenum and other portions of the proximal small intestine) of fats into monoglycerides, glycerol and free fatty acids, proteins into peptides and amino acids, and starch into 
dextrins and short chain sugars. Enzepi was demonstrated to be safe and effective in patients with CF and pancreatic insufficiency, showing significant improvements in the coefficient of fat absorption and coefficient of nitrogen absorption [52, 53]. However, Enzepi was withdrawn from the market in July 2017 for commercial reasons [54].

\section{Trends in CF drug development}

$\mathrm{CF}$ is a rare disease and therefore sponsors developing potential new medicines for this therapeutic area may apply for the status of orphan drugs at the EMA [55].

Applications for orphan designation are examined by the EMA's Committee for Orphan Medicinal Products (COMP), whose members are experts representing all EU member states. Sponsors who obtain orphan designation benefit from a number of incentives, including the possibility to apply for national and European grants for clinical development (within the Horizon 2020 programme), EMA protocol assistance, and 10 years market exclusivity in relation to similar products once the orphan medicine is on the market.

To qualify for orphan designation, a medicinal product must meet the following criteria: 1) it must be intended for the treatment, prevention or diagnosis of a disease that is life threatening or chronically debilitating; 2) the prevalence of the condition in the EU must not be more than 5 in 10000 or it must be unlikely that marketing of the medicinal product would generate sufficient returns to justify the investment needed for its development; and 3) no satisfactory method of diagnosis, prevention or treatment of the condition concerned can be authorised, or, if such an authorised method exists, the medicinal product must be of significant benefit to those affected by the condition.

Orphan designation is granted after request from the sponsor and positive evaluation by the COMP. Medicinal products are usually at an early stage of development (nonclinical and/or early clinical) at the time of initial orphan designation. For products that are evaluated in a marketing authorisation procedure, their orphan status needs to be confirmed by COMP, including the demonstration of significant benefit with respect to the approved products for the same condition. In the case of lack of confirmation of orphan status at the time of marketing authorisation, a medicinal product can still be centrally authorised but loses the 10 years of market exclusivity linked to the orphan status.

Between 2001 and 2016, 57 products received an orphan designation and at present only eight of these products have completed their clinical trial development and been granted a marketing authorisation from the EMA. Out of these eight products, only four (Cayston, TOBI Podhaler, Bronchitol and Kalydeco) kept their orphan status at the time of marketing authorisation (table 2). The other authorised products were withdrawn by the Community Register of designated orphan medicinal products at the request of the sponsor, after significant benefit was questioned by the COMP at the time of marketing authorisation. Orphan status was never requested for Enzepi because its therapeutic indication also included more common conditions such as chronic pancreatitis, post-pancreatectomy or pancreatic cancer.

Supplementary table A gives a good representation of the general trend in CF drug development over time, while figure 1 classifies the 57 designated orphan products, based on their mode of action. The majority (74\%) act on the symptoms of the disease, with anti-inflammatory compounds (22\%) and antibiotics $(21 \%)$ being the most frequent classes of medicinal products, followed by drugs acting on airways clearance (17\%) and antibacterials (14\%). Enzyme replacement therapy for fat regulation represents $3 \%$ of the overall designated orphan drugs, while the DNA plasmid developed by Imperial Innovations Ltd represents the only orphan drug for CFTR gene therapy. The remaining products belong to the class of ion transport modulators. Nine of them (16\%) act exclusively on the CFTR protein and are therefore called CFTR modulators, while the remaining three (5\%) act on other ion channels (e.g. sodium or chloride) and are defined in figure 1 as $\mathrm{Na}^{+} / \mathrm{Cl}^{-}$transport modulators.

The discovery of Kalydeco triggered a boost in the search for novel compounds acting as modulators of the mutated protein (supplementary table A).

From 2008 to 2016, the CFTR modulators represented 23\% of the designated orphan products compared with $8 \%$ in the period 2001-2007. However, it is important to highlight that within the nine CFTR modulators which received orphan designation, four of them (ivacaftor (Kalydeco), lumacaftor, tezacaftor (i.e. VX-661) and ivacaftor/lumacaftor (Orkambi)) were developed by the same company (Vertex Pharmaceuticals, Abingdon, UK). Lumacaftor received an orphan designation in 2010 and the fixed-dose combination lumacaftor/ivacaftor received orphan designation in 2014, which was subsequently withdrawn at the time of marketing authorisation.

Other CFTR modulators include 4,4',6-trimethylangelicin (TMA) and two correctors: 8-cyclopentyl-1,3-dipropylxanthine and 2-(7-ethoxy-4-(3-fluorophenyl)-1-oxophthalazin-2(1H)-yl)- $N$ methyl- $N$-(2-methylbenzo[d]oxazol-6-yl) acetamide (FDL-169). TMA combines corrector and potentiator 


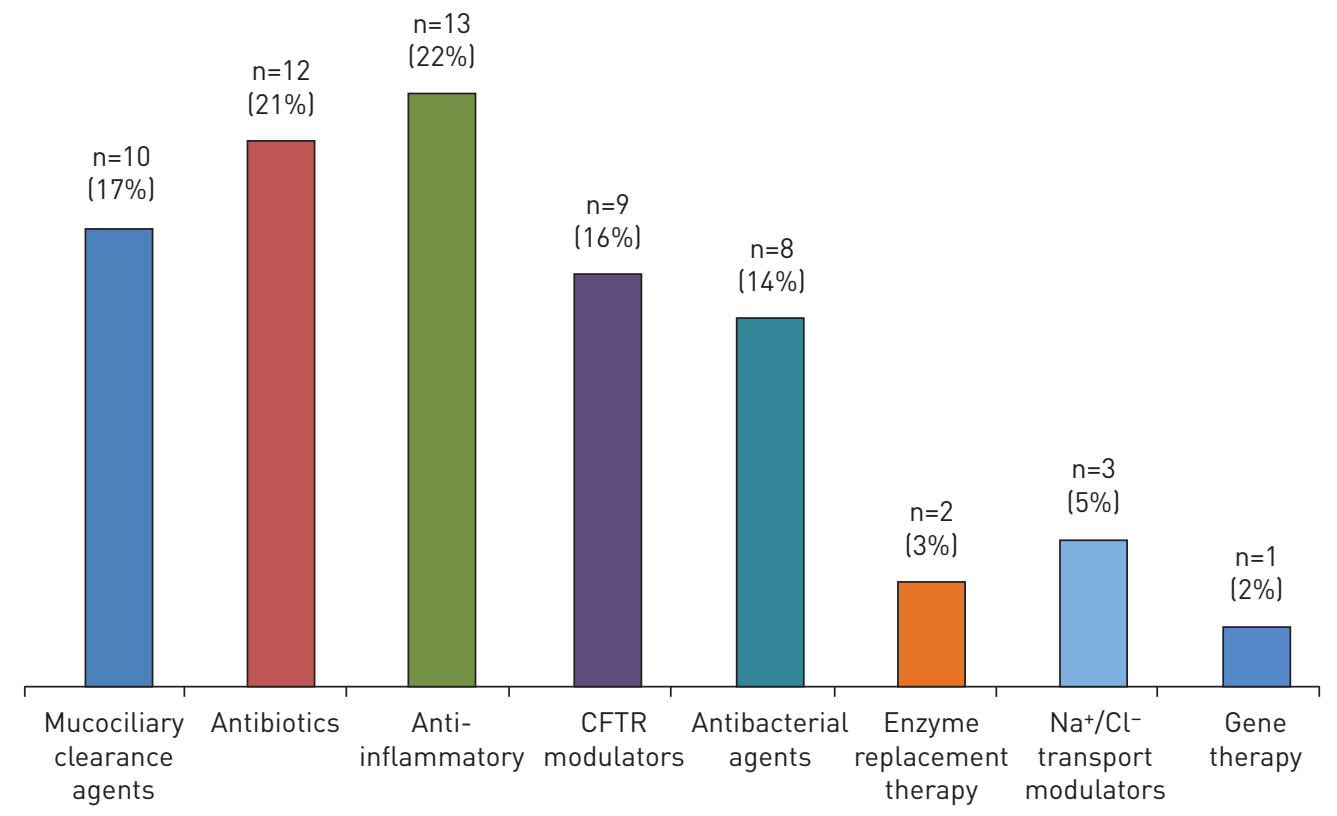

FIGURE 1 Classification by type of cystic fibrosis (CF) products that received orphan designation during the period 2001-2016. CFTR: CF transmembrane conductance regulator. 4,4',6-trimethylangelicin was counted twice in the histogram, being both a CFTR modulator and an anti-inflammatory agent.

activity, together with anti-inflammatory activity. Additional treatments designed to restore CFTR function are QR-010 and ataluren. The antisense nucleotide QR-010 (developed by ProQR, Leiden, The Netherlands) targets the F508del mutation, but in contrast to correctors that act on mutant CFTR protein, it binds to the CFTR mRNA thus preventing its translation. Instead, ataluren (developed by PTC Therapeutics, Dublin, Ireland) acts exclusively on nonsense mutations, which generate the termination codons UAA, UAG or UGA and result in a premature termination of polypeptide synthesis during translation. This drug candidate was intended to promote read-through of premature termination codons by inserting an amino acid and allowing full-length CFTR protein synthesis. However, a recent pivotal phase 3 clinical trial of ataluren in patients living with nonsense mutation CF failed to meet primary and secondary end-points, resulting in the withdrawal of the marketing authorisation application under the EMA centralised procedure $[56,57]$.

\section{Analysis of nonclinical end-points in the light of the clinical results of CFTR modulators}

Nonclinical studies are often used by sponsors as proof of concept to demonstrate medical plausibility for the intended indication and preliminary efficacy in the frame of orphan designation. In fact, out of 57 designated orphan products, 27 received the designation based only on pre-clinical data. This indicates that pre-clinical studies presented in the successful applications were considered to have the potential of anticipating effects and clinical relevance of new products in CF treatment.

Published data for ivacaftor, lumacaftor and Orkambi report three pre-clinical end-points used to monitor CFTR function and therefore evaluate the efficacy of the aforementioned products [50, 51, 58]. A summary of the published studies performed, including the type of cells and the associated mutations, is given in table 3.

The first end-point takes into account the total ionic current (IT) due to the cell surface channel density, gating activity and conductance. This end-point was measured with the use of the Ussing chamber technique and was applied to Fischer rat thyroid (FRT) cells bearing the G551D or F508del mutations or primary human bronchial epithelial (HBE) cells from CF patients bearing the F508del or G551D/F508del CFTR mutations.

The second end-point is the CFTR open channel probability $(P O)$, which represents the fraction of time that a single CFTR protein channel is open and transports ions. The PO was measured on NIH-3T3 cells bearing the G551D or F508del mutations.

The third end-point is CFTR maturation, which relies on Western blotting techniques and monitors the cellular trafficking of CFTR to the apical surface. This study, performed on both FRT and HBE cells 
TABLE 3 Nonclinical end-points used in different cell lines to demonstrate efficacy of ivacaftor, lumacaftor and orkambi

\begin{tabular}{lcccc} 
Nonclinical end-point & Cell mutation & Ivacaftor & Lumacaftor & Orkambi \\
\hline Total ionic current ( $\boldsymbol{H}$ ) (FRT cells) & G551D & $\checkmark$ & & \\
& F508del & $\checkmark$ & $\checkmark$ \\
Total ionic current ( $\boldsymbol{H}$ ) (HBE cells) & F508del & $\checkmark$ & $\checkmark$ \\
Open probability (Po) (NIH-3T3 cells) & G551D/F508del & $\checkmark$ & $\checkmark$ & $\checkmark^{\#}$ \\
CFTR maturation (FRT cells) & G551D & $\checkmark$ & $\checkmark$ & \\
CFTR maturation (HBE cells) & F508del & $\checkmark$ & $\checkmark$
\end{tabular}

IT: total ionic current; FRT: Fischer rat thyroid; HBE: human bronchial epithelial; Po: open channel probability; CFTR: cystic fibrosis transmembrane conductance regulator. " : type of cells not reported.

bearing the F508del mutation, is especially relevant for the two products acting as correctors (i.e. lumacaftor and Orkambi).

Looking at table 3, a direct comparison of the three products can only be achieved for IT in HBE cells bearing the F508del mutation and for the PO assay in NIH-3T3 cells. In vitro studies using CF HBE cells are reported to be the most adequate in vitro model to test the pharmacological action of CFTR modulators, given that these cells exhibit many of the morphological and functional characteristics believed to be associated with CF in the lungs, including low chloride transport, excessive sodium transport, defective fluid regulation and decreased cilia beating [59].

Table 4 shows the results obtained from the in vitro studies (e.g. PO and IT) for ivacaftor, lumacaftor and Orkambi. In terms of $P O$, the three products were able to fully restore the CFTR gating activity for both mutations (results not shown) [50, 51, 58]. However, in terms of $I \mathrm{~T}$ measurement using $\mathrm{HBE}$ cells, lumacaftor and Orkambi managed to restore the functioning of the F508del CFTR protein in a range of

TABLE 4 Recovery of cystic fibrosis transmembrane conductance regulator (CFTR) functionality from nonclinical studies, and forced expiratory volume in $1 \mathrm{~s}$ (FEV1) and sweat chloride results from clinical trials for ivacaftor, lumacaftor and orkambi

\begin{tabular}{|c|c|c|c|c|c|}
\hline Product & $\begin{array}{l}\text { CFTR mutation in } \\
\text { HBE cells in } \\
\text { nonclinical } \\
\text { studies }\end{array}$ & $\begin{array}{l}\text { Recovery of CFTR } \\
\text { functionality } \\
\text { compared with } \\
\text { wild-type } \%\end{array}$ & $\begin{array}{l}\text { CFTR mutation in } \\
\text { patients for } \\
\text { clinical trials }\end{array}$ & $\begin{array}{l}\text { FEV1 \% } \\
\text { pred } \\
\text { change }\end{array}$ & 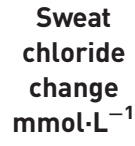 \\
\hline \multirow[t]{2}{*}{ Ivacaftor } & $\begin{array}{l}\text { Homozygous } \\
\text { F508del }\end{array}$ & $<10$ & Homozygous F508del & $1.7^{\pi}$ & $-2.9^{+}$ \\
\hline & G551D/F508del & 48 & G551D/x mutation ${ }^{\#}$ & $10.5^{\S}$ & $-48.1^{f}$ \\
\hline Lumacaftor & $\begin{array}{l}\text { Homozygous } \\
\text { F508del }\end{array}$ & 14 & Homozygous F508del & $0.47^{\# \#}$ & $-8.2^{\text {ๆा }}$ \\
\hline Orkambi & $\begin{array}{l}\text { Homozygous } \\
\text { F508del }\end{array}$ & 25.1 & Homozygous F508del & $3.3^{++}$ & $-9.5^{\S \S}$ \\
\hline
\end{tabular}

HBE: human bronchial epithelial. " : patients that have the G551D mutation on at least one allele (the " $x$ " stands for the G551D mutation or any other class I-V mutation [23]); ": absolute change in FEV $1 \%$ pred from baseline through week 16 between ivacaftor and placebo groups (the observed change was not statistically significant) $[60] ;{ }^{+}$: sweat chloride reduction in the ivacaftor versus placebo groups from baseline through week 16 [60]; ${ }^{\S}$ : increase from baseline in FEV $1 \%$ pred through week 48 in the ivacaftor group compared with placebo [23]; ${ }^{f}$ : sweat chloride reduction in the ivacaftor versus placebo groups from

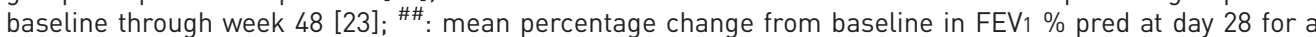
$200 \mathrm{mg}$ dose [61]; ${ }^{\text {กा: }}$ mean treatment difference in sweat chloride from baseline (-placebo) at day 28 for a $200 \mathrm{mg}$ dose [61]; ${ }^{++}$: treatment difference obtained from the pooled data of two phase 3 pivotal studies for the average absolute change from baseline in $\mathrm{FEV} 1 \%$ pred at week 16 and 24 (dose: lumacaftor $600 \mathrm{mg}$ / ivacaftor $250 \mathrm{mg}$ ) [58]; $\S \S$ : treatment difference in sweat chloride from a phase 2 trial for the lumacaftor $600 \mathrm{mg}$ once daily/ivacaftor $250 \mathrm{mg}$ once every $12 \mathrm{~h}$ homozygous group compared with the pooled combination placebo group in the mean absolute change from baseline at day 56 [58]. 
14-25.1\% with respect to the wild-type enzyme. Within this type of assay, ivacaftor showed the lowest and highest efficacy, with a recovery of the mutant channel activity of $<10 \%$ [62] and 48\% [50] for the homozygous F508del and G551D/F508del mutations, respectively.

Genotype-to-phenotype studies that correlate in vivo CFTR function with disease severity suggest that a recovery of the CFTR function $>10 \%$ based on chloride transport would lead to a milder CF pathology $[63,64]$. The milder disease is characterised by a lower incidence of pancreatic insufficiency, later age at diagnosis, a more moderate lung function decline and lower sweat chloride levels $\left(\sim 80 \mathrm{mmol} \cdot \mathrm{L}^{-1}\right)$ compared with those with minimal CFTR chloride transport (i.e. patients with F508del homozygous CF). By analysing the outcome from the clinical trials performed with the three products (table 4), a correlation cannot be established for all the products between the in vitro efficacy data and the results based on the clinical end-points of the phase $2 / 3$ studies (FEV1 and sweat chloride). Of the three compounds, ivacaftor demonstrated a significant efficacy both in the in vitro studies using HBE cells from patients with the G551D/F508del mutation and in the clinical studies performed on patients with at least one G551D mutation. In this case a stable increase of $\sim 10 \%$ from baseline of the FEV 1 levels and a significant decrease in sweat chloride $\left(48.1 \mathrm{mmol} \cdot \mathrm{L}^{-1}\right)$ compared with placebo at 48 weeks were observed [23].

The CFTR recovery data for the F508del mutation shows that, while a good correlation can be observed for ivacaftor, where $<10 \%$ recovery of CFTR activity translated into a nonstatistical significant increase for FEV 1 (1.7\%) [60,62], a 14\% CFTR recovery for lumacaftor did not translate into a significant clinical efficacy, in terms of both FEV1 and sweat chloride [61]. The combination product, Orkambi, which showed a very promising $25.1 \%$ recovery of CFTR functionality, demonstrated only a modest increment in FEV1 [57]. This data questions the validity of a possible correlation between a milder CF pathology and a CFTR activity $>10 \%$. These results also highlight the need for developing alternative in vitro and/or in vivo pre-clinical models to better predict the clinical efficacy of these new agents. Recently, the US Food and Drug Administration extended the label of Kalydeco to patients aged $\geqslant 2$ years who have at least one of 23 residual function mutations in the CFTR gene and based its decision, in part, on the results of laboratory testing, which it used in conjunction with evidence from earlier clinical trials. The same approach in Europe would have to be endorsed by the Committee of Human Medicinal Products if the company applied for such extension [65].

Concerning the search of alternative nonclinical models, a promising method based on organoids has been recently developed [66-68]. With this method, airway and intestinal epithelial cells are cultured within a gel, where they form spheroid structures with a fluid-filled lumen. Importantly, CFTR activity results in fluid secretion into the lumen, thus causing organoid swelling. Therefore, the responsiveness of mutant CFTR to pharmacological modulators is easily evaluated by measuring the extent of swelling. A clear advantage of working with organoids, particularly those of intestinal origin, is the possibility to start the culture with a minimal number of cells collected with a biopsy. The high proliferating ability of intestinal cells allows generation of a large number of organoids that can be used for functional assays. CFTR residual activity data obtained from organoids and the response to pharmacological CFTR modulators are well correlated with the intestinal current measurements done on rectal biopsies, an established method to measure CFTR function [69]. Interestingly, the response to lumacaftor and ivacaftor of organoids from donors with identical CFTR mutations has been reported to be variable, indicating that the individual genetic background plays a role in the response to drug therapy [70]. Large datasets can be generated within weeks from organoid cultures expressing different CFTR genotypes, allowing measurement of CFTR residual activity and response to CFTR modulators for individual patient's samples. Such tests would be impossible with more conventional methods such as intestinal current measurement as a single evaluation can be done per each biopsy. Of particular importance is the assessment of the correlation between organoid readout and clinical response to treatments. In a recent study, the different response to ivacaftor of organoids from two patients with rare CFTR mutations was well correlated with the efficacy of this drug in vivo [70]. Although the first results of in vitro-in vivo correlation of responses to CFTR modulators in rectal organoids are encouraging, larger studies are required to better define the translational potential of this approach. This notwithstanding, the characterisation of CFTR modulators in rectal organoids represents an important step towards personalised therapy in CF.

\section{Conclusions and recommendations}

$\mathrm{CF}$ is the most common autosomal recessive disease among Caucasians, and is a systemic disease showing variable severity and a broad clinical spectrum depending on the target organs and systems affected. Although many organs are affected in CF, pulmonary disease is the major cause of morbidity and mortality.

The quest of new medicines for CF has contributed to a better understanding of the condition, to a refinement of its taxonomy and did indeed open new knowledge pathways for the development of disease modifiers. 
However, the relatively slow progress in developing new therapies for CF and the lack of clinical relevant efficacy in some recent clinical confirmatory studies indicate, inter alia, some limitations in the translatability of the current pre-clinical and in vitro models. More robust models and pre-clinical end-points along with appropriate genotype-to-phenotype studies for a better identification of clinical predictors are needed to successfully move new molecules from the pre-clinical to the clinical setting and increase the prediction of the clinical impact for new promising CF products.

A collaborative effort among patient organisations, academic research centres and networks [71], industry, and private and public investors is required to expand the knowledge base using modern life science tools including, for instance, organoids, novel in vivo and in silico models digitalisation, and big data science.

Early interaction with regulators is recommended to establish a continued dialogue and guidance on how to satisfactorily address issues in product development making the best use of the EU innovation offices network [72] and following the pathways available in Europe to progress novel methodologies [73] and innovative medicines $[74,75]$ for the benefit of patients and society at large.

Author contribution: The views expressed in this article are the personal views of the authors and may not be understood or quoted as being made on behalf of or reflecting the position of the agencies or organisations with which the authors are affiliated.

Conflict of interest: F. Bertozzi has patents IT 102017000028127 and IT 102017000028184 pending. T. Bandiera has patents IT 102017000028127 and IT 102017000028184 pending. L.J.V. Galietta has patents IT 102017000028127 and IT 102017000028184 pending.

\section{References}

Elborn JS. Cystic fibrosis. Lancet 2016; 388: 2519-2531.

Riordan JR. CFTR and prospects for therapy. Annu Rev Biochem 2008; 77: 701-726.

Farrel PM. The prevalence of cystic fibrosis in the European Union. J Cyst Fibros 2008; 7: 450-453.

McCormick J, Mehta G, Olesen HV, et al. Comparative demographics of the European cystic fibrosis population: a cross-sectional database analysis. Lancet 2010; 375: 1007-1013.

Liu F, Zhang Z, Csanády L, et al. Molecular structure of the human CFTR ion channel. Cell 2017; 169: 85-95.

Riordan JR. Assembly of functional CFTR chloride channels. Annu Rev Physiol 2005; 67: 701-718.

Kim Chiaw P, Eckford PD, Bear CE. Insights into the mechanisms underlying CFTR channel activity, the molecular basis for cystic fibrosis and strategies for therapy. Essays Biochem 2011; 50: 233-248.

8 Lukacs GL, Verkman AS. CFTR: folding, misfolding and correcting the $\Delta$ F508 conformational defect. Trends $\mathrm{Mol}$ Med 2012; 18: 81-91.

9 Lubamba B, Dhooghe B, Noel S, et al. Cystic fibrosis: insight into CFTR pathophysiology and pharmacotherapy. Clin Biochem 2012; 45: 1132-1144.

10 Zielenski J. Genotype and phenotype in cystic fibrosis. Respiration 2000; 67: 117-133.

11 Farrell PM, Rosenstein BJ, White TB, et al. Guidelines for diagnosis of cystic fibrosis in newborns through older adults: Cystic Fibrosis Foundation consensus report. J Pediatr 2008; 153: S4-S14.

12 Okiyoneda T, Lukacs GL. Fixing cystic fibrosis by correcting CFTR domain assembly. J Cell Biol 2012; 199: 199-204.

13 Stoltz DA, Meyerholz DK, Welsh MJ. Origins of cystic fibrosis lung disease. N Engl J Med 2015; 372: 1574-1575.

14 Boucher RC. New concepts of the pathogenesis of cystic fibrosis lung disease. Eur Respir J 2004; 23: 146-158.

15 Davis PB. Cystic fibrosis since 1938. Am J Respir Crit Care Med 2006; 173: 475-482.

16 SickKids. Cystic Fibrosis Mutation Database. www.genet.sickkids.on.ca Date last accessed: Aug 19, 2017. Date last updated: April 25, 2011.

17 Welsh MJ, Smith AE. Molecular mechanisms of CFTR chloride channel dysfunction in cystic fibrosis. Cell 1993; 73: 1251-1254.

18 Rowe SM, Miller S, Sorscher EJ. Cystic fibrosis. N Engl J Med 2005; 352: 1992-2001.

19 Lukacs GL, Chang XB, Bear C, et al. The delta F508 mutation decreases the stability of cystic fibrosis transmembrane conductance regulator in the plasma membrane. Determination of functional half-lives on transfected cells. J Biol Chem 1993; 268: 21592-21598.

20 Wilschanski M, Zielenski J, Markiewicz D, et al. Correlation of sweat chloride concentration with classes of the cystic fibrosis transmembrane conductance regulator gene mutations. J Pediatr 1995; 127: 705-710.

21 Du K, Sharma M, Lukacs GL. The DeltaF508 cystic fibrosis mutation impairs domain-domain interactions and arrests post-translational folding of CFTR. Nat Struct Mol Biol 2005; 12: 17-25.

22 Rogan MP, Stoltz DA, Hornick DB. Cystic fibrosis transmembrane conductance regulator intracellular processing, trafficking, and opportunities for mutation-specific treatment. Chest 2011: 139: 1480-1490.

23 Ramsey BW, Davies J, McElvaney NG, et al. A CFTR potentiator in patients with cystic fibrosis and the G551D mutation. N Engl J Med 2011; 365: 1663-1672.

24 Okiyoneda T, Barrière H, Bagdány $\mathrm{M}$, et al. Peripheral protein quality control removes unfolded CFTR from the plasma membrane. Science 2010; 329: 805-810.

25 Barrett PM, Alagely A, Topol EJ. Cystic fibrosis in an era of genomically guided therapy. Hum Mol Genet 2012; 21: R66-R71.

26 De Boeck K, Wilschanski M, Castellani C, et al. Cystic fibrosis: terminology and diagnostic algorithms. Thorax 2006; 61: 627-635.

27 Quinton PM. Role of epithelial $\mathrm{HCO}_{3-}$ transport in mucin secretion: lessons from cystic fibrosis. Am J Physiol Cell Physiol 2010; 299: C1222-C1233.

28 Pezzulo AA, Tang XX, Hoegger MJ, et al. Reduced airway surface $\mathrm{pH}$ impairs bacterial killing in the porcine cystic fibrosis lung. Nature 2012; 487: 109-113. 

fibrosis after newborn screening. J Pediatr 2009; 155: 623-628.

screening. Am J Respir Crit Care Med 2008; 178: 1238-1244.

31 Castellani $\mathrm{C}$ Southern KW, Brownlee $\mathrm{K}$, et al. European best practice guidelines for cystic fibrosis neonatal screening. J Cyst Fibros 2009; 8: 153-173.

32 Sinaasappel M, Stern M, Littlewood J, et al. Nutrition in patients with cystic fibrosis: a European consensus. J Cyst Fibros 2002; 1: 51-75.

33 Andersen DH. Cystic fibrosis of the pancreas and its relation to celiac disease: a clinical and pathological study. Am J Dis Child 1938; 56: 344-399.

34 Burgel PR, Bellis G, Olesen HV, et al. Future trends in cystic fibrosis demography in 34 European countries. Eur Respir J 2015; 46: 133-141.

35 Wilschanski M. Novel therapeutic approaches for cystic fibrosis. Discov Med 2013; 15: 127-133.

36 Ikpa PT, Bijvelds MJ, de Jonge HR. Cystic fibrosis: toward personalized therapies. Int J Biochem Cell Biol 2014; 52: $192-200$.

37 European Medicines Agency. Cayston European public assessment report. www.ema.europa.eu/ema/index.jsp? curl=pages/medicines/human/medicines/000996/human_med_000686.jsp\&mid=WC0b01ac058001d124 Date last accessed: Aug 31, 2017. Date last updated: Aug 31, 2017.

38 Parkins MD, Elborn JS. Aztreonam lysine: a novel inhalational antibiotic for cystic fibrosis. Expert Rev Respir Med 2010; 4: 435-444.

39 Fiel SB. Aerosolized antibiotics in cystic fibrosis: current and future trends. Expert Rev Respir Med 2008; 2: 479-487.

40 Fiel SB. Aerosolized antibiotics in cystic fibrosis: an update. Expert Rev Respir Med 2014; 8: 305-314.

41 Klinger-Strobel M, Lautenschläger C, Fischer D, et al. Aspects of pulmonary drug delivery strategies for infections in cystic fibrosis - where do we stand? Expert Opin Drug Deliv 2015; 12: 1-24.

42 Geller DE. Aerosol antibiotics in cystic fibrosis. Respir Care 2009; 54: 658-670.

43 Ryan G, Singh M, Dwan K. Inhaled antibiotics for long-term therapy in cystic fibrosis. Cochrane Database Syst Rev 2011; 3: CD001021.

44 Daviskas E, Anderson SD, Brannan JD, et al. Inhalation of dry-powder mannitol increases mucociliary clearance. Eur Respir J 1997; 10: 2449-2454.

45 Bilton D, Robinson P, Cooper P, et al. Inhaled dry powder mannitol in cystic fibrosis: an efficacy and safety study. Eur Respir J 2011; 38: 1071-1080.

46 Aitken ML, Bellon G, De Boeck K, et al. Long-term inhaled dry powder mannitol in cystic fibrosis - an international randomized study. Am J Respir Crit Care Med 2012; 185: 645-652.

47 Anderson P. Emerging therapies in cystic fibrosis. Ther Adv Respir Dis 2010; 4: 177-185.

48 Lee TWR, Matthews DA, Blair GE. Novel molecular approaches to cystic fibrosis therapy. Biochem J 2005; 387: $1-15$.

49 Pettit RS, Fellner C. CFTR modulators for the treatment of cystic fibrosis. P T 2014; 39: 500-511.

50 Van Goor F, Hadida S, Grootenhuis PD, et al. Rescue of CF airway epithelial cell function in vitro by a CFTR potentiator, VX-770. Proc Natl Acad Sci USA 2009; 106: 18825-18830.

51 Van Goor F, Hadida S, Grootenhuis PD, et al. Correction of the F508del-CFTR protein processing defect in vitro by the investigational drug VX-809. Proc Natl Acad Sci USA 2011; 108: 18843-18848.

52 Wooldridge JL, Heubi JE, Amaro-Galvez R, et al. EUR-1008 pancreatic enzyme replacement is safe and effective in patients with cystic fibrosis and pancreatic insufficiency. J Cyst Fibros 2009; 8: 405-417.

53 Taylor CJ, Thieroff-Ekerdt R, Shiff S, et al. Comparison of two pancreatic enzyme products for exocrine insufficiency in patients with cystic fibrosis. J Cyst Fibros 2016; 15: 675-680.

54 European Medicines Agency. Withdrawal of the marketing authorisation in the European Union for Enzepi. 2017. www.ema.europa.eu/docs/en_GB/document_library/Public_statement/2017/07/WC500232416.pdf Date last accessed: Sept 14, 2017.

55 European Medicines Agency. Orphan designation. www.ema.europa.eu/ema/index.jsp?curl=pages/regulation/ general/general_content_000029.jsp\&mid=WC0b01ac0580b18a41 Date last accessed: Aug 19, 2017. Date last updated: July 24, 2017.

56 European Medicines Agency. Withdrawal of the application for a change to the marketing authorisation for Translarna (ataluren). 2017. www.ema.europa.eu/docs/en_GB/document_library/Medicine_QA/2017/03/ WC500224384.pdf Date last accessed: Aug 19, 2017.

57 PTC Therapeutics. PTC Therapeutics announces results from pivotal phase 3 clinical trial of ataluren in patients living with nonsense mutation cystic fibrosis [press release]. 2017. http://ir.ptcbio.com/releasedetail.cfm? releaseid=1015471 Date last accessed: Aug 19, 2017.

58 European Medicines Agency. Orkambi assessment report. 2015. www.ema.europa.eu/docs/en_GB/document library/EPAR _ _Public assessment_report/human/003954/WC500197613.pdf Date last accessed: Aug 19, 2017.

59 Neuberger T, Burton B, Clark H, et al. Use of primary cultures of human bronchial epithelial cells isolated from cystic fibrosis patients for the pre-clinical testing of CFTR modulators. Methods Mol Biol 2011; 741: 39-54.

60 Flume PA, Liou TG, Borowitz DS, et al. Ivacaftor in subjects with cystic fibrosis who are homozygous for the F508del-CFTR mutation. N Engl J Med 2012; 142: 718-724.

61 Clancy JP, Rowe SM, Accurso FJ, et al. Results of a phase IIa study of VX-809, an investigational CFTR corrector compound, in subjects with cystic fibrosis homozygous for the F508del-CFTR mutation. Thorax 2012; 67: 12-18.

62 European Medicines Agency. Kalydeco assessment report. 2012. www.ema.europa.eu/docs/en_GB/document library/EPAR_-_Public_assessment_report/human/002494/WC500130766.pdf Date last accessed: Jan 15, 2018.

63 Strausbaugh SD, Davis PB. Cystic fibrosis: a review of epidemiology and pathobiology. Clin Chest Med 2007; 28: 279-288.

64 Rowe SM, Accurso F, Clancy JP. Detection of cystic fibrosis transmembrane conductance regulator activity in early-phase clinical trials. Proc Am Thorac Soc 2007; 4: 387-398.

65 Food and Drug Administration. FDA expands approved use of Kalydeco to treat additional mutations of cystic fibrosis [press release]. 2017. www.fda.gov/NewsEvents/Newsroom/PressAnnouncements/ucm559212.htm Date last accessed: Jan 17, 2018. 
Barkauskas CE, Chung MI, Fioret B, et al. Lung organoids: current uses and future promise. Development 2017; 144: 986-997.

67 Boj SF, Vonk AM, Statia M, et al. Forskolin-induced swelling in intestinal organoids: an in vitro assay for assessing drug response in cystic fibrosis patients. J Vis Exp 2017; (120): e55159.

68 Dekkers JF, van der Ent CK, Beekman JM. Novel opportunities for CFTR-targeting drug development using organoids. Rare Dis 2013; 1: e27112.

69 Dekkers JF, Wiegerinck CL, de Jonge HR, et al. A functional CFTR assay using primary cystic fibrosis intestinal organoids. Nat Med 2013; 19: 939-945.

70 Dekkers JF, Berkers G, Kruisselbrink E, et al. Characterizing responses to CFTR-modulating drugs using rectal organoids derived from subjects with cystic fibrosis. Sci Transl Med 2016; 8: 344ra84.

71 European Medicines Agency. European Network of Paediatric Research at the European Medicines Agency (Enpr-EMA). www.ema.europa.eu/ema/index.jsp?curl=pages/partners_and_networks/general/general_content_ 000303.jsp\&mid=WC0b01ac05801df74a Date last accessed: March 14, 2018. Date last updated: Sept 8, 2017.

72 European Medicines Agency. Innovation in medicines. www.ema.europa.eu/ema/index.jsp?curl=pages/regulation/ general/general_content_000334.jsp\&mid=WC0b01ac05800bald9 Date last accessed: Aug 19, 2017. Date last updated: Aug 9, 2017.

73 European Medicines Agency. Qualification of novel methodologies for medicine development. www.ema.europa. eu/ema/index.jsp?curl=pages/regulation/document_listing/document_listing_000319.jsp\&mid=WC0b01ac0580022bb0 Date last accessed: Aug 19, 2017. Date last updated: July 24, 2017.

74 European Medicines Agency. PRIME: priority medicines. www.ema.europa.eu/ema/index.jsp?curl=pages/regulation/ general/general_content_000660.jsp\&mid=WC0b01ac05809f8439 Date last accessed: Aug 19, 2017. Date last updated: July 27,2017

75 European Medicines Agency. Scientific advice and protocol. assistance. www.ema.europa.eu/ema/index.jsp? curl=pages/regulation/general/general_content_000049.jsp\&mid=WC0b01ac05800229b9 Date last accessed: Aug 19, 2017. Date last updated: Aug 21, 2017. 